\title{
Additions and Corrections
}

Vol. 31 (2008) 1199-1208

Combined Effects of an 3-Hydroxy-3-Methylglutaryl Coenzyme A Reductase Inhibitor and Angiotensin II Receptor Antagonist on Nitric Oxide Bioavailability and Atherosclerotic Change in Myocardial Infarction-Prone Watanabe Heritable Hyperlipidemic Rabbits

Toshio Imanishi, Hideyuki Ikejima, Atsushi Tsujioka, Akio Kuroi, Katsunobu Kobayashi, Masashi Shiomi, Yasuteru Muragaki,

Seiichi Mochizuki, Masami Goto, Kiyoshi Yoshida, and Takashi Akasaka

Page 1199: Author's name

The author name "Atsushi Tsujioka" should be replaced to "Hiroto Tsujioka." 